\title{
Research Paper \\ Comparison of Static and Dynamic Balance of Athletes of Different Sports in Conditions With and Without Posture Disturbances
}

\author{
Mostafa Shadkanlu Ostad ${ }^{1}\left(\mathbb{0}, *^{*}\right.$ Aliasghar Norasteh ${ }^{2}$ (), Hamed Babagoltabar Samakoush ${ }^{1}$ (C)
}

1. Department of Sport Management, Faculty of Physical Education, University of Guilan, Rasht, Iran.

2. Department of Sport Physiology, Faculty of Physical Education, University of Guilan, Rasht, Iran.

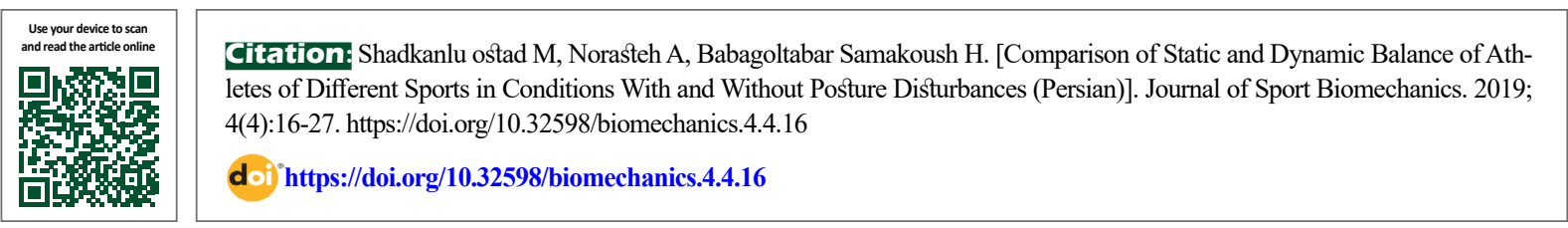

\section{(i) \$}

Article Info:

Received: 13 Oct 2018

Accepted: 05 Jan 2019

Available Online: 01 Mar 2019

Key words:

Static balance, Dynamic balance, Dual-task, Attention, Wrestling, Football, Karate

\begin{abstract}
Objective: Balance is a key component of motor skills to maintain posture and perform complex sports skills. The aim of this study was to compare the static and dynamic balance of athletes in different sports using different conditions of with/without postural perturbation.

Methods: Participants were 42 athletes with 3 years of sports experience assigned into three groups of wrestlers $(n=14)$, football players $(n=14)$ and karate players $(n=14)$. Static balance was assessed using the Balance Error Scoring System and dynamic balance measurement was done using the Star Excursion Balance Test. For measurement of postural perturbations or cognitive task, the countdown technique was used. Data were analyzed using Analysis of variance (ANOVA), Analysis of Covariance (ANCOVA), and paired t-test at a significance level of $P \leq 0.05$.

Results: There was no significant difference between the static and dynamic balance of athletes with postural perturbation in three groups. No significant difference was found between static balance with and without postural perturbation in three groups, but the difference was significant different between dynamic balance with and without postural perturbation, where their dynamic balance without postural perturbation was better.
\end{abstract}

Conclusion: Coaches and physiotherapists are recommended to use dual-task techniques in assessments.

\section{Extended Abstract}

\section{Introduction}

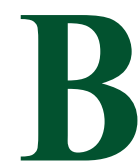

alance is a critical component of motor skills to maintain posture and perform complex exercise skills. Studies using the dual-task paradigm have reported that postural control requires considerable resources. Cognition refers to a range of high-level brain functions, including the ability to learn and remember information, solve problems, concentrate, maintain and divide attention, understand, use language, and correctly understand the environment.

Attention, as a cognition's aspect, significantly impacts postural control. For example, a football player knows that when shooting, the gravity line moves forward; thus, to maintain balance, he pushes the center of gravity backward. In basketball, the athletes throw their Body Mass (BM) forward to throw the ball, followed by the center of gravity to move forward and out of the supporting surface; thus, one has to take a step forward to avoid falling.

* Corresponding Author:

Aliasghar Norasteh, PhD.

Address: Department of Sport Physiology, Faculty of Physical Education, University of Guilan, Rasht, Iran.

Tel: +98 (912) 3896962

E-Mail: asgharnorasteh@yahoo.com 
Postural control requires coordination between the sensory, motor, and cognitive systems. Furthermore, the dysfunction of each component affects postural maintenance. Attention is the individuals' information processing capacity. To study the effect of cognitive systems on balance control, researchers have evaluated the rate of human balance control dependence on attention. The present study compared the static and dynamic balance in different sports athletes with and without postural disturbances.

\section{Participants and Methods}

The study subjects consisted of 14 wrestlers (Mean \pm SD of age: $19.85 \pm 2.79 \mathrm{y} ;$ Mean \pm SD of height: $177.14 \pm 5.17$ $\mathrm{cm}$; Mean \pm SD of weight: $71.64 \pm 1.32 \mathrm{~kg}$; BM index: $22.81 \pm 3.43 \mathrm{~kg} / \mathrm{m}^{2}$ ), 14 soccer players (Mean \pm SD of age: $21.57 \pm 2.02 \mathrm{y} ; \mathrm{Mean} \pm \mathrm{SD}$ of height: $178.86 \pm 6.58 \mathrm{~cm}$; Mean \pm SD of weight: $74.35 \pm 8.02 \mathrm{~kg}$; BM index $23.20 \pm 1.60$ $\mathrm{kg} / \mathrm{m}^{2}$ ), and 14 karatekas (Mean \pm SD of age: $21.35 \pm 1.54$ $\mathrm{y}$; Mean \pm SD of height: $178.07 \pm 4.10 \mathrm{~cm}$; Mean \pm SD of weight: $76.92 \pm 4.98 \mathrm{~kg}$; BM index: $24.32 \pm 1.76 \mathrm{~kg} / \mathrm{m}^{2}$ ).

Bureau of Indian Standards (BIS) and Star tests were used to evaluate static and dynamic balance, respectively. To perform the cognitive task in this study, the reverse numbering of random numbers was used. The cognitive activity consisted of reverse coding, starting from a random number selected. For example, a digit between 200-300 out of 30 numbers introduced by the examiner, by counting down by 7 . Then, as the test started, the subject counted down and reduced the number by 7 in his mind. After 30 seconds, the subject was requested to report the last number. He was requested to engage his mind in numerical calculation entirely. The number of times a person was able to count down 7 numbers could not be accurately calculated; therefore, the final answer was considered in terms of being true or false. The obtained data, after a descriptive evaluation, were analyzed using the Analysis of Variance (ANOVA), Analysis of Covariance (ANCOVA), and t-test at $\mathrm{P}<0.05$ (Table $1 \& 2$ ).

\section{Results}

Data analysis suggested that static and dynamic balance preservation in wrestlers, soccer players, and karatekas was not significantly different. Furthermore, static and dynamic balance preservation associated with postural disturbances was not significantly different in these groups. There was no significant difference in static balance without postural disturbances, compared to static balance with postural disturbances. There was a significant difference in dynamic balance with no postural disturbances, compared to dynamic balance with postural disturbances. It was also better to maintain dynamic balance without postural disturbances.

\section{Discussion}

Researchers have recently used the dual-task paradigm to assess the effect of cognitive factors on postural control in evaluating balance control. Therefore, this study aimed to compare the static and dynamic balance of different sports athletes with and without postural disturbances. The collected results indicated that the karatekas had the lowest

Table 1. One-way ANOVA results for the comparison of static and dynamic balance with and without cognitive task in the study subjects

\begin{tabular}{|c|c|c|c|}
\hline Test & Sport & $\mathbf{F}$ & Sig. \\
\hline \multirow{3}{*}{ Static balance without a cognitive task (s) } & Wrestling & \multirow{3}{*}{2.057} & \multirow{3}{*}{0.142} \\
\hline & Soccer & & \\
\hline & Karate & & \\
\hline \multirow{3}{*}{ Static balance with a cognitive task (s) } & Wrestling & \multirow{3}{*}{0.430} & \multirow{3}{*}{0.653} \\
\hline & Soccer & & \\
\hline & Karate & & \\
\hline \multirow{3}{*}{ Dynamic balance without a cognitive task ( $\mathrm{cm} /$ foot length) } & Wrestling & \multirow{3}{*}{2.057} & \multirow{3}{*}{0.142} \\
\hline & Soccer & & \\
\hline & Karate & & \\
\hline \multirow{3}{*}{ Dynamic balance with the cognitive task (cm/foot length) } & Wrestling & \multirow{3}{*}{0.554} & \multirow{3}{*}{0.579} \\
\hline & Soccer & & \\
\hline & Karate & & \\
\hline
\end{tabular}


Table 2. Correlated t-test results for comparing static and dynamic balance with and without cognitive task in the three study groups

\begin{tabular}{cccc}
\hline Sport & \multicolumn{1}{c}{ Test } & t & Sig. \\
\hline \multirow{2}{*}{ Wrestling } & Static balance with cognitive task & -1.022 & 0.326 \\
& Dynamic balance with the cognitive task & 2.387 & 0.03 \\
Soccer & Static balance with cognitive task & 0.751 & 0.466 \\
& Dynamic balance with cognitive task & 2.469 & 0.02 \\
Karate & Static balance with cognitive task & -1.876 & 0.08 \\
& Dynamic balance with cognitive task & 12.288 & 0.001 \\
\hline
\end{tabular}

mean number of errors in the BIS test and the highest mean length of maturity in the star test. However, the wrestlers had the lowest mean maturity in the star test. Data analysis revealed no significant difference in the static and dynamic balance between the studied wrestlers, soccer players, and karatekas.

Recent studies suggested a significant necessity for postural control. Moreover, these requirements vary depending on the postural function, age, and the balance ability of individuals. The current study compared the static and dynamic balance of different sports athletes with and without postural disturbances. In all three exercise groups, the extent of foot entrapment in dynamic balance decreased with a cognitive task. As a result, they are more involved with motions; thus, they require higher dynamic balance and moving to maintain balance, i.e. focus on skills and motions. Accordingly, athletes should perform their skill and balance exercises through cognitive tasks.

\section{Conclusion}

Based on the study results, it is suggested that trainers and physiotherapists use a dual-task paradigm in their evaluations.

\section{Ethical Considerations}

Compliance with ethical guidelines

All subjects in the present study participated with full consent and were aware of all stages of the research. Also aware of the confidentiality of their personal information, they had complete authority to exit any stages of the investigation.

\section{Funding}

The present paper was extracted from the MSc thesis of Mostafa Shadkanlou of the Department of Corrective Exercise and sport Injuries of the University of Guilan and it didn't use any financial backing.

\section{Authors' contributions}

Conceptualization, methodology: Mostafa Shadkanlu Ostad, Aliasghar Norasteh; Resources, writing - original draft preparation: Hamed Babagoltabar Samakoush; Writing - review \& editing: Hamed Babagoltabar Samakoush, Aliasghar Norasteh; Visualization, supervision, project administration: Aliasghar Norasteh.

\section{Conflicts of interest}

In this paper, there is no conflict of interest. 


\title{
مقايسه تعادل ايستا و يوياى ورزشكاران رشتههاى مختلف ورزشى در شرايط با و بدون اعمال اغتشاشات ياسجر تعادر ايستا
}

مصطفى شادكانلو استاد' هـ، "على اصغر نورسته' (ه)، حامد باباكل تبار سماكوش' (ه)

ا I. كارشئاسارشد تربيت بدنى، دانشكده تربيتبدنى و علوم ورزشى، دانشكاه كيلان، رشت، ايران.

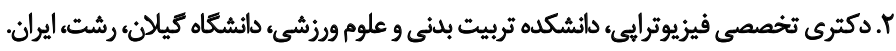

\begin{abstract}
حكيد

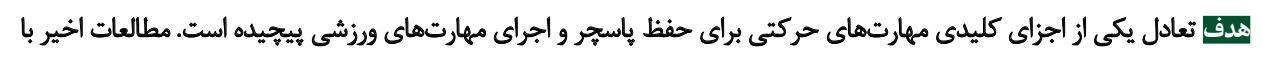

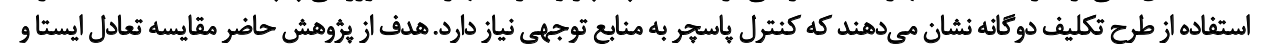

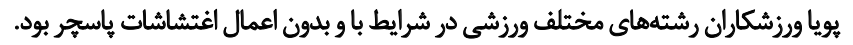

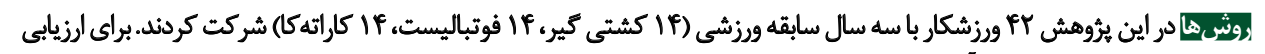

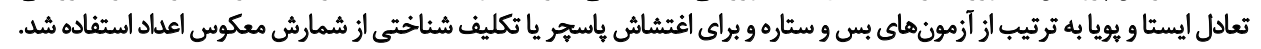

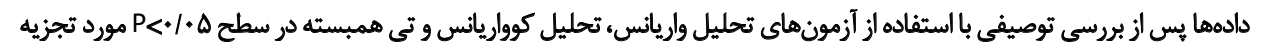
و تحليل آمارى قرار كرفتند.

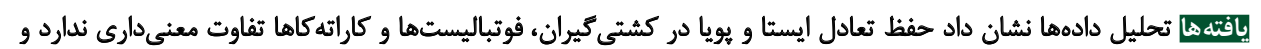

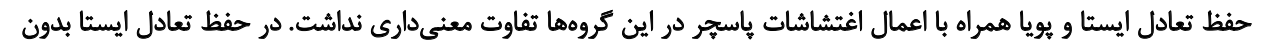

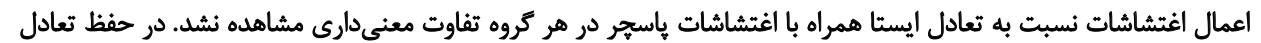

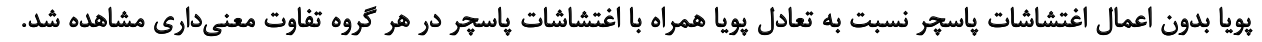

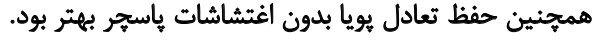

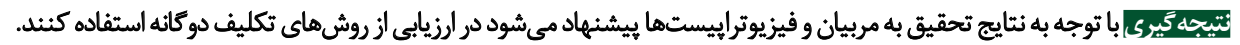

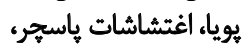

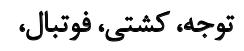
كاراته

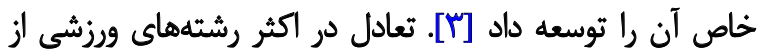

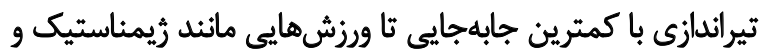

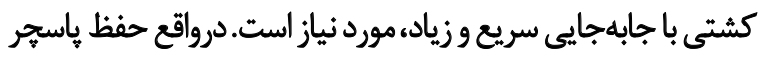

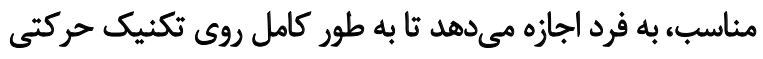

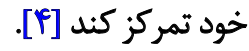

حفظ راستايستادن به كنترل خوب وضعيت و اندازه حركت مركز

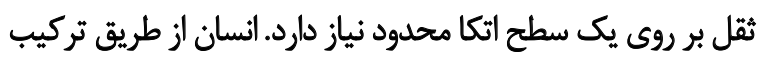

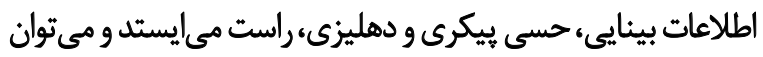

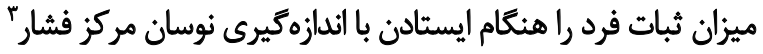

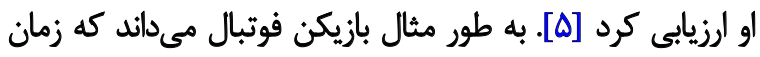

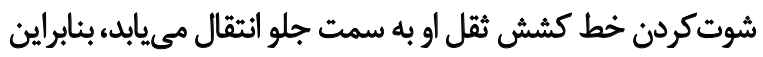

dotes

2. Dynamic balance

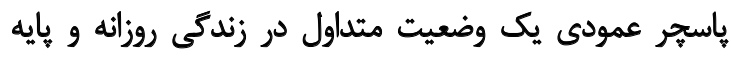

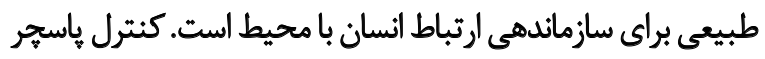

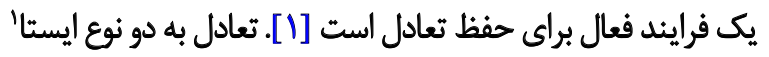

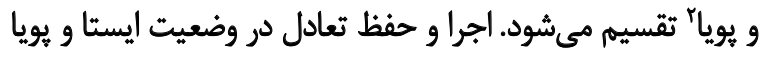

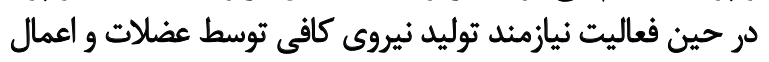

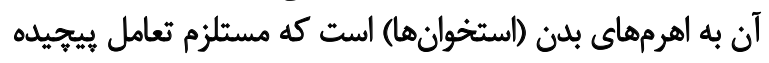

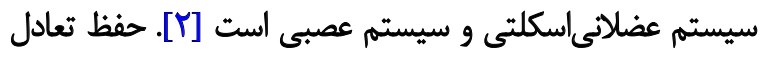

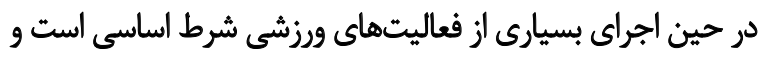
يكى از عوامل آمادكى جسمانى است كه مي توان به به وسيله تمرينات 
نسبت ورزشكار غيرحرفهاي داشتند [919]. از طرف ديكر مارتين

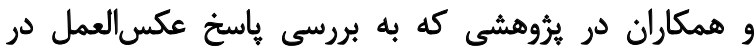

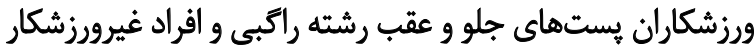

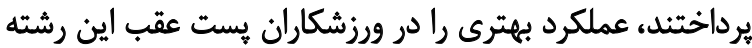

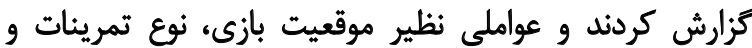

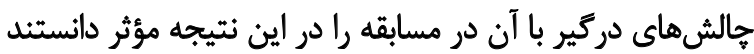

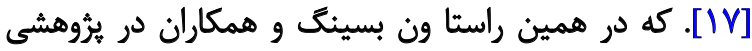

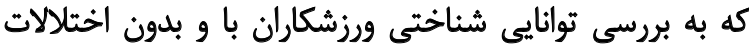

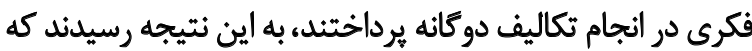

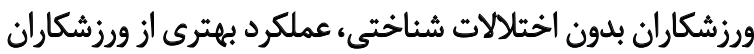

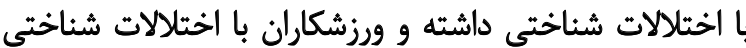

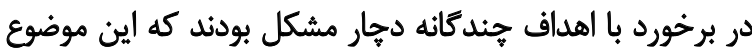

نشاندهنده اهميت ادراك شناختى در عملكرد است [IA] در تحقيق ديكرى كه لودر و همكاران به مقايسه تمرينات

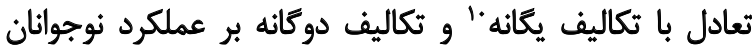

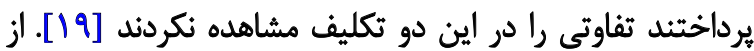

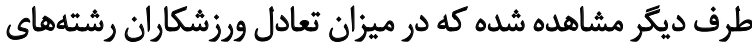

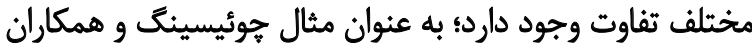

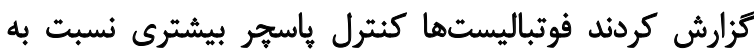

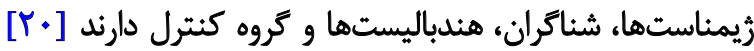

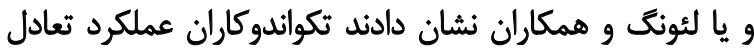

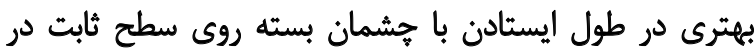

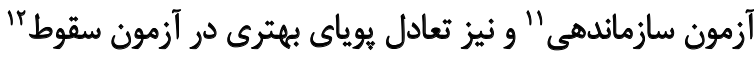

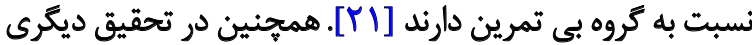

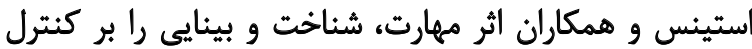

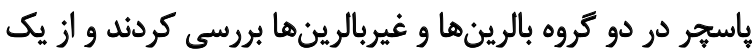

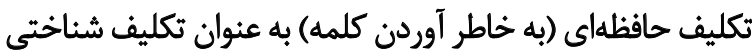

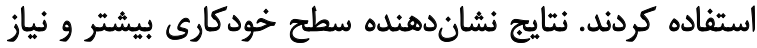

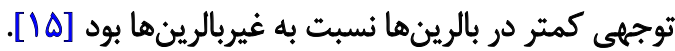
با توجه به مطالب ذكرشده در زمينه ادراك شناختى و اهميت

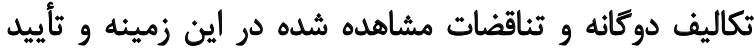

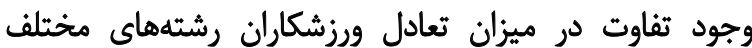

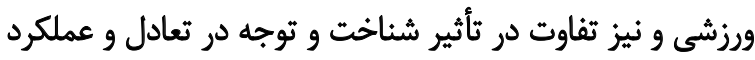

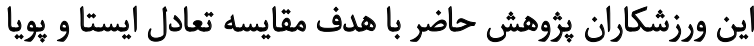

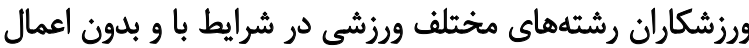

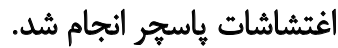

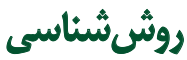

آزمودنىهاى اين تحقيق را كليه كشتى كيران، فوتباليستها و ورئ

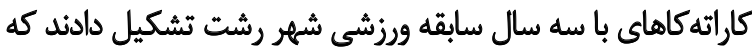

10. Single-Task Balance

11. Sensory organization test (SOT)

12. Drop test
براي ازدستندادن تعادل، مركز ثقل خود را به سمت عقب ميبرد.

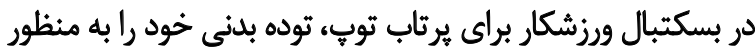

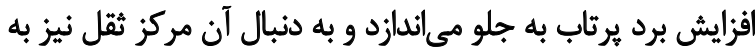

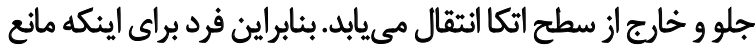

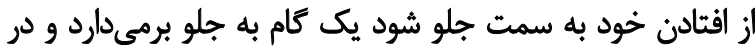

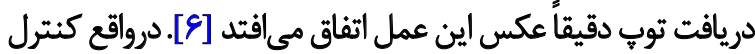

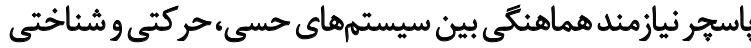

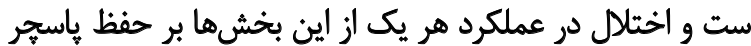
فرد اثر مى كذارد [V]

شناخت" به محدودهاى از عملكردهاى سطوح بالاى مغزى اطلاق

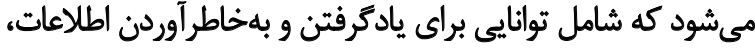

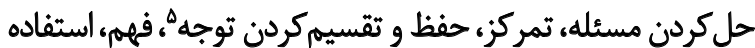

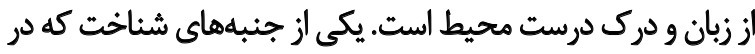
كنترل ياسجر نقش دارد توجه است [A] توجه، ظرفيت بردازش اطلاعات يك فرد است. براى مطالعه در

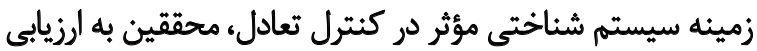

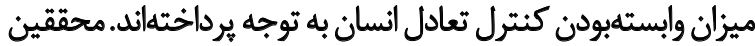

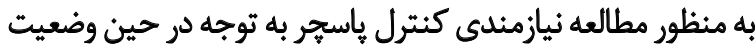

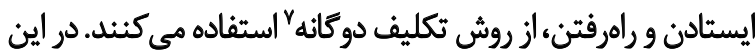

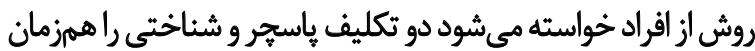

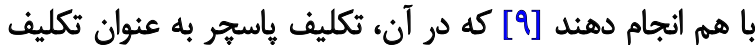

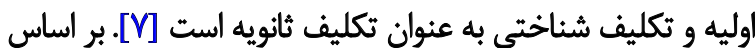

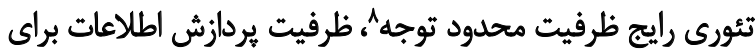

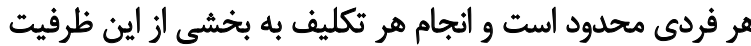

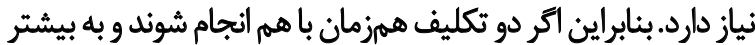

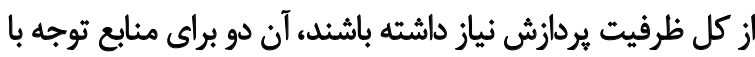

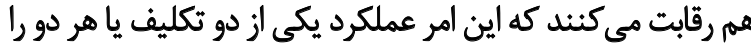

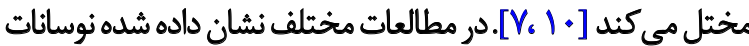

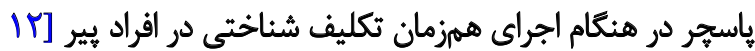

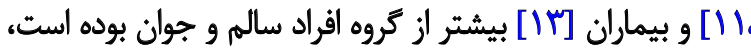

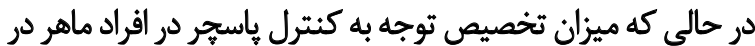

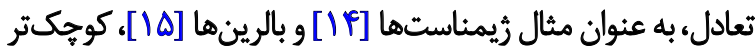

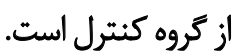

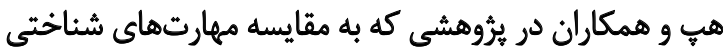

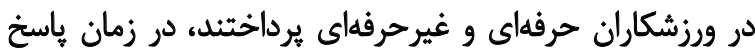

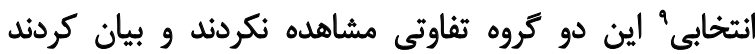

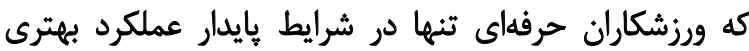
4. Cognition
5. Divided Attention
6. Attention
7. Dual-Task Paradigm
8. Limited Capacity Theory of Attention
9. Choice response time 


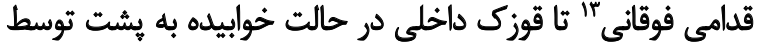

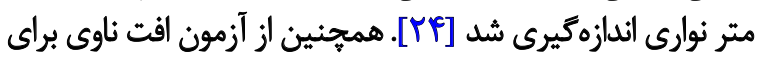

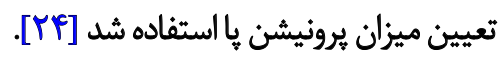

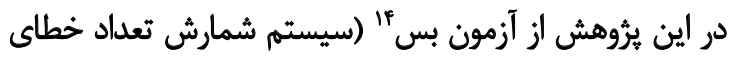

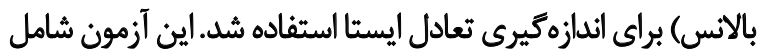

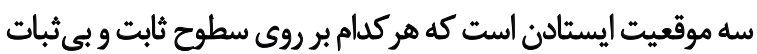

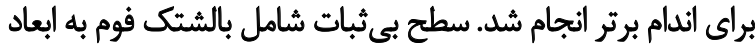

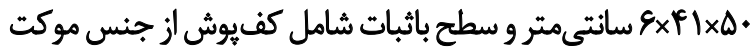

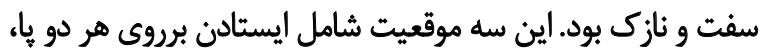

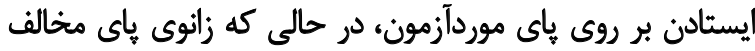

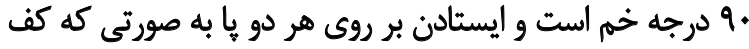

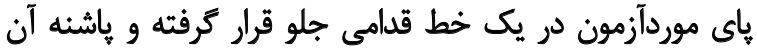

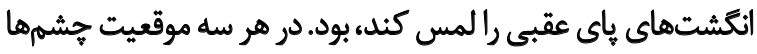

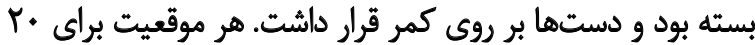

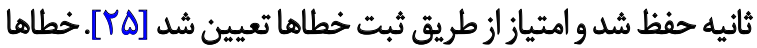

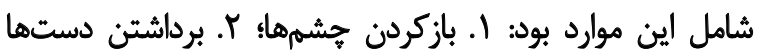

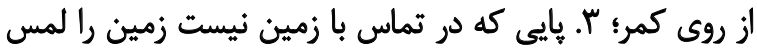

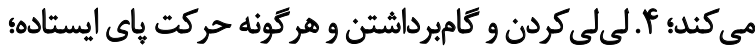

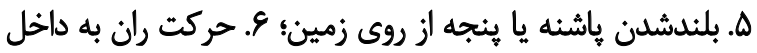

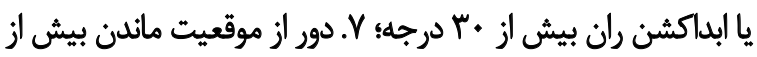

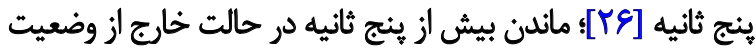
استاندارد، (تصوير شماره ) (1). ارزيابي تعادل يويا در اين ثيروهش با استفاده از آزمون ستاره

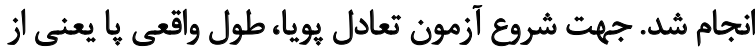

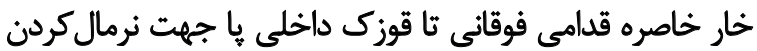

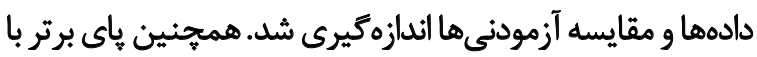

13. Anterior superior iliac spine (ASIS)

14. Balance Error Scoring System (BESS)

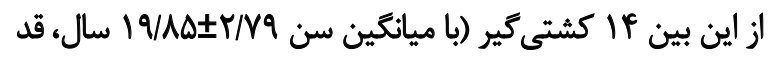
IVV/If $\pm \Delta / / V$

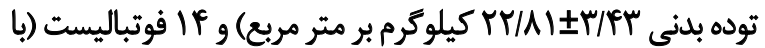

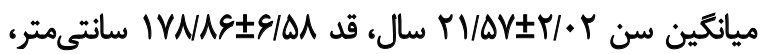

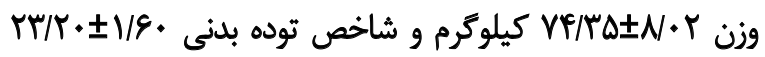

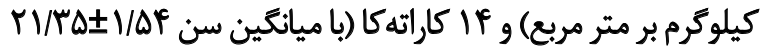
سال، قد • •

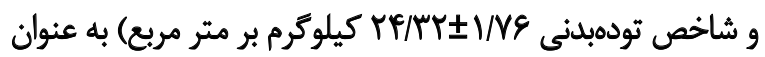
نمونههاى تحقيق و به صورت غير تصادفى هدف دار انتخاب شديند براى جمعآورى اطلاعات جمعيتشناختى و اطلاعات در مورد

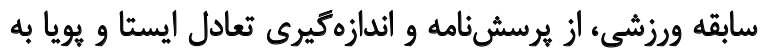

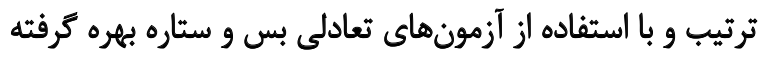

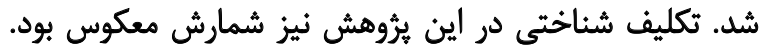

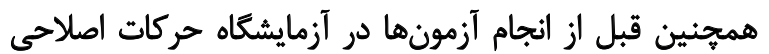

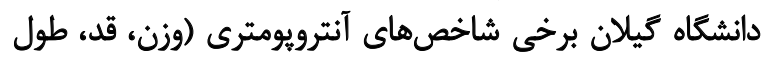

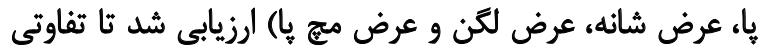

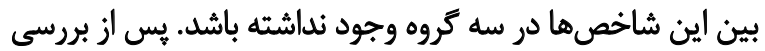

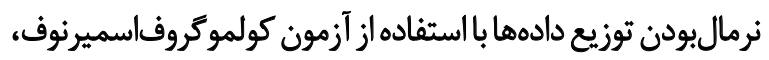

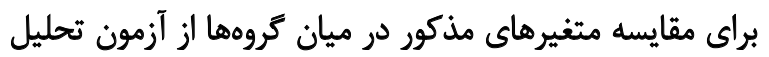

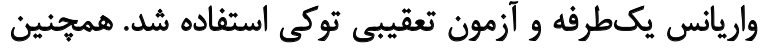

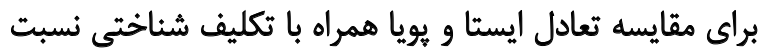

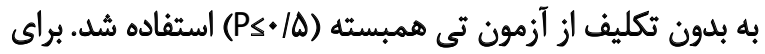

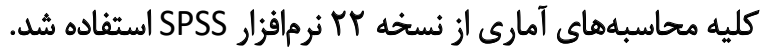
اندازهيرى طول كف ها در وضعيت عدم تحمل وزن، به حالت

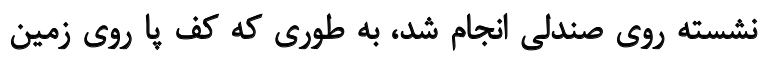

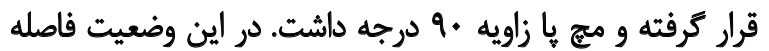

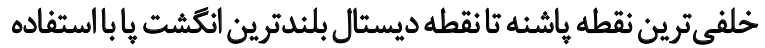

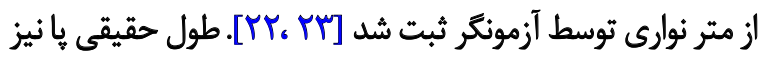

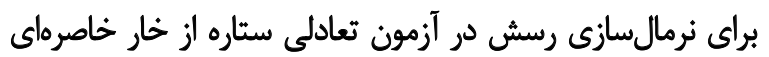


در هر جهت به صورت جداكانه، از فرمول شماره الستفاده شد

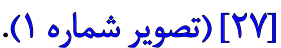

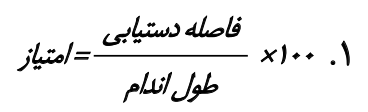

همجنين در اين بُروهش جهت انجام تكليف شناختى در اين

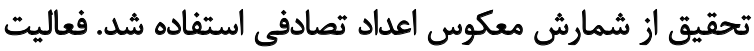

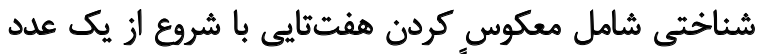

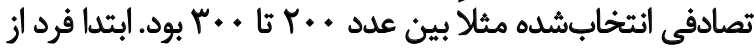

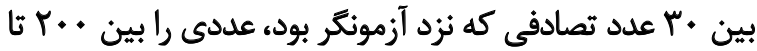

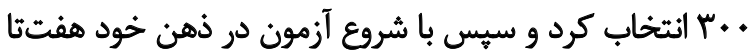
هفتا از آن كم مى كرد. يس إز اتمام •ب ثانيه از فرد خوراسته
استفاده از آزمون شوت توب تعيين شد. براى انجام آزمون ستاره،

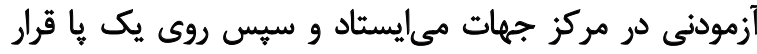

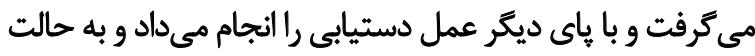

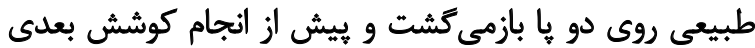

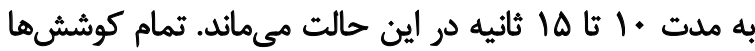

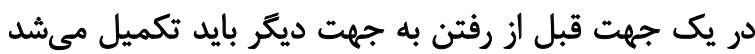

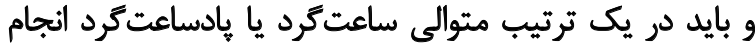

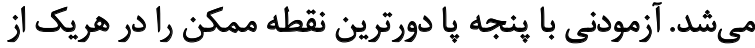

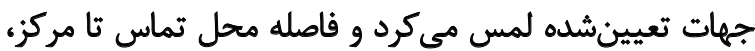

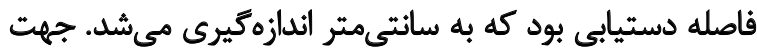
بهدست آوردن اختلاف بين ميانكين نمرات تعادل (آزمون ستاره)

جدول ا. نتايج آزمون تحليل كوواريائس جهت بررسي اثر متغيرهاى مداخله كر بر تعادل ايستاو يويا در آزمودنيها

\begin{tabular}{|c|c|c|c|c|c|}
\hline sig. & Df & $\mathbf{F}$ & متغير وابسته & ميانكيندانحراف معيار & متغير مستقل \\
\hline$. / 9 F \mid$ & 1 & .1 .08 & تعادل ايستا & \multirow{3}{*}{$r \cdot / 9 r \pm r / e r$} & \multirow{3}{*}{ سن (سال) } \\
\hline & & & & & \\
\hline .1988 & 1 & $\cdot 1 \cdot+r$ & تعادل يويا & & \\
\hline.$/ r q$. & 1 & $1 / 7 / 9$ & تعادل ايستا & \multirow{3}{*}{$n \in / \mu+ \pm 9 / 4 r$} & \multirow{3}{*}{ ونن (كيلوكرم) } \\
\hline & & & & & \\
\hline - AMPA & 1 & $1 \cdot P D$ & تعادل يويا & & \\
\hline.$/ T \Delta F$ & 1 &.$/ 945$ & تعادل ايستا & \multirow{3}{*}{$m / \mu f \pm r / \mu f$} & \multirow{3}{*}{ (كيلوكرم بر متر مربع) BMI } \\
\hline & & & & & \\
\hline . ART & 1 & $\cdot 1 \cdot \mathrm{H}$ & تعادل يويا & & \\
\hline . /TAF & 1 & l/fel & تعادل ايستا & \multirow{3}{*}{$I V N \cdot r \pm \Delta / r q$} & \multirow{3}{*}{ قد (سانتىمتر) } \\
\hline & & & & & \\
\hline.$/ 914$ & 1 & $\cdot 1 \cdot 11$ & تعادل يويا & & \\
\hline$\cdot \mid A \cdot A$ & 1 & $\cdot|8|$ & تعادل ايستا & \multirow{3}{*}{ Q)/EE $\mathrm{r} / \Delta \mathrm{V}$} & \multirow{3}{*}{ طول با (سايتىىت) } \\
\hline & & & & & \\
\hline .1 .08 & 1 & $r / r$. & تعادل يويا & & \\
\hline . KeP & 1 & I/rege & تعادل ايستا & \multirow{3}{*}{$r \cdot / 9 f \pm r / 1 f^{f}$} & \multirow{3}{*}{ عرض شائه (سانتىمتر) } \\
\hline & & & & & \\
\hline . / 990 & 1 & $.1 \cdot v$ & تعادل يويا & & \\
\hline .1198 & 1 & //AAQ & تعادل ايستا & \multirow{3}{*}{ rNEq $\pm 1 / F T$} & \multirow{3}{*}{ عرض لكن (سانتيمتر) } \\
\hline & & & & & \\
\hline.$/ m 11$ & 1 & $1 / 111$ & تعادل يويا & & \\
\hline.$/ 290$ & 1 & . paq & تعادل ايستا & \multirow{3}{*}{$N \cdot 1 \pm \cdot / 48$} & \multirow{3}{*}{ عرض محج يا (سانتىمتر) } \\
\hline & & & & & \\
\hline - /per & 1 &.$/ 81 F$ & تعادل يويا & & \\
\hline.$/ r v q$ & 1 & /Ar. & تعادل ايستا & \multirow{3}{*}{$V / F E \pm .109$} & \multirow{3}{*}{ افت ناوى (ميلىمترى) } \\
\hline & & & & & \\
\hline . /Far & 1 & .104 & تعادل يويا & & \\
\hline . Mer & 1 & IT/ & تعادل ايستا & \multirow{3}{*}{$r \& / \& \Delta \pm 1 / . r$} & \multirow{3}{*}{ طول كف پا (سانتىمتر) } \\
\hline & & & & & \\
\hline . & 1 & . & تعادل يويا & & \\
\hline
\end{tabular}

مجله بيومكانيك ورنش 
جدول r. نتايج آزمون تحليل واريانس يكراهه براي مقايسه تعادل ايستا و يويا با و بلون تكليف شناختى در آزمودئىها

\begin{tabular}{|c|c|c|c|c|c|}
\hline sig. & Df & $\mathbf{F}$ & ميانكين土|نحراف معيار & كيروه ورزشى & آزآمون \\
\hline.$/ N T$ & $r$ & $Y / \Delta V$ & 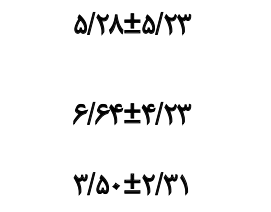 & كشتى فوتبال & تكليف شناختي (ثانيها \\
\hline . IqAT & $r$ &.$/ p r$. & 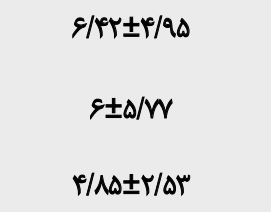 & كشتى كوتبال & تعادل ايستا با تكليف \\
\hline . /NT & $r$ & $r / \Delta V$ & 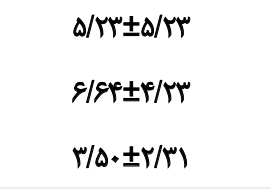 & كشتى & تعادل يويا بلدون تكليف \\
\hline ./AV & $r$ & - IDAF & 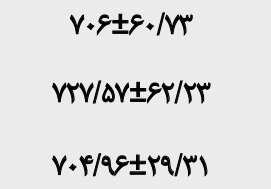 & كشتى & 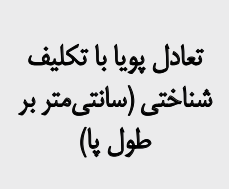 \\
\hline
\end{tabular}

مجله بيومكانيك ورنش

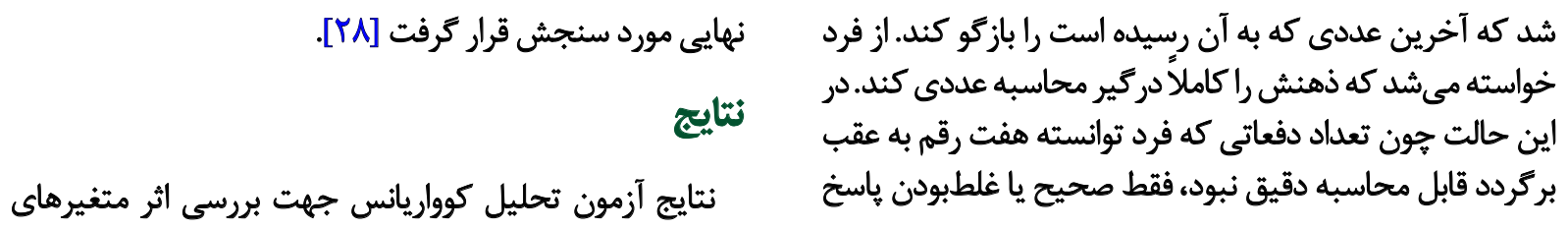

جدول ب. نتايج آزمون تى همبسته براى مقايسه تعادل ايستاو ويويا با و بدون تكليف شناختى در سه كروه آزمودنى

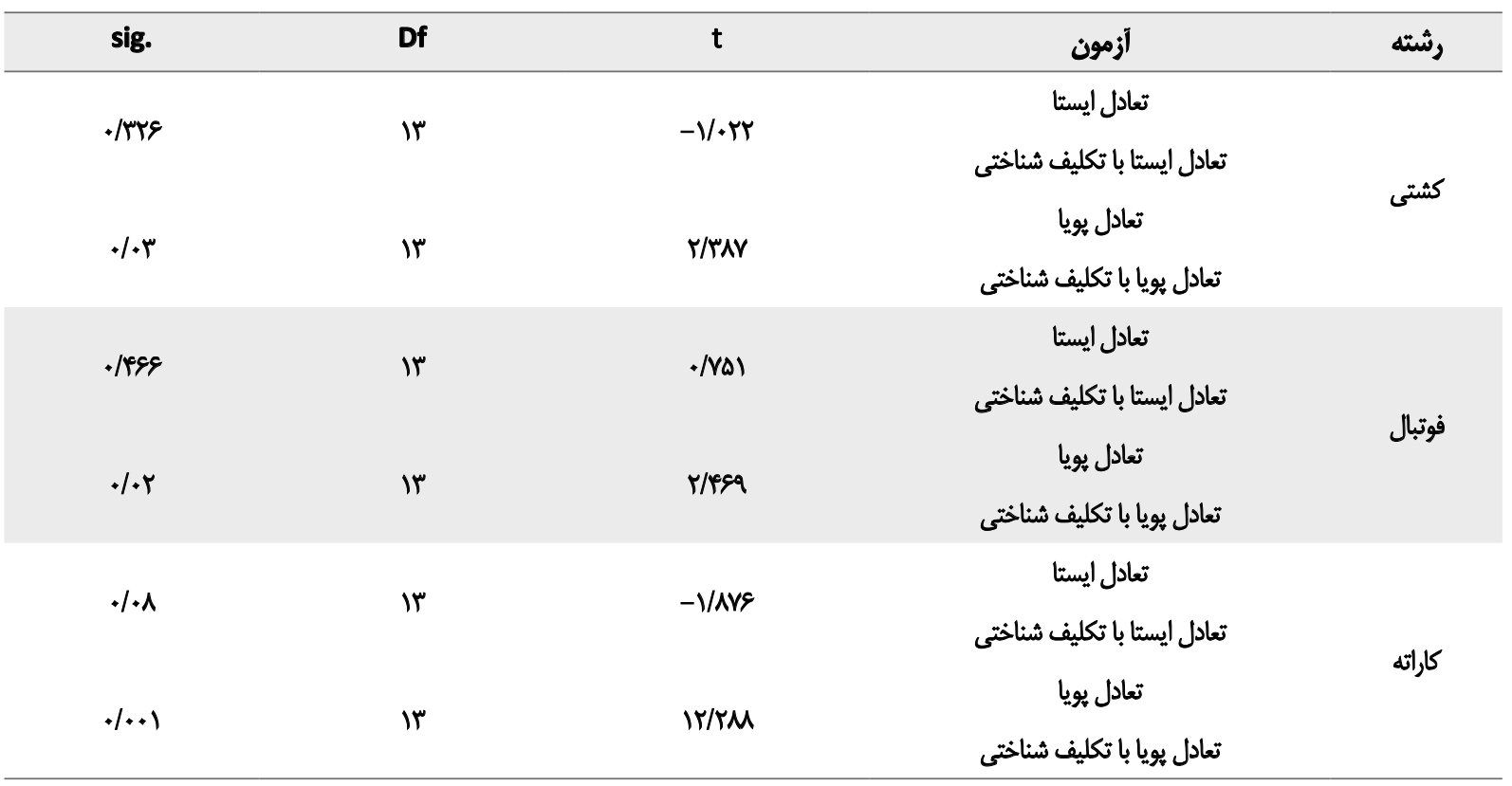

مجله بيومكانيك ولنش 


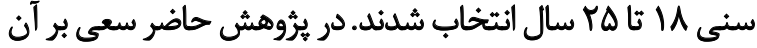

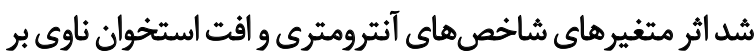

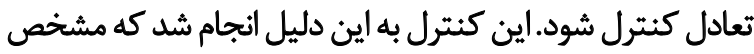

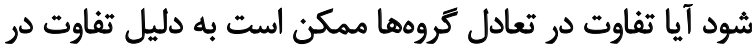

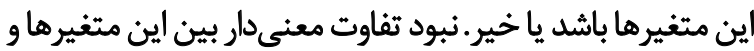

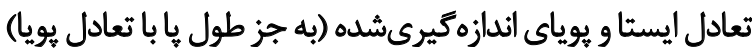

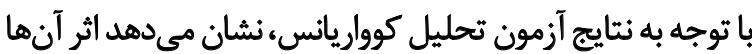

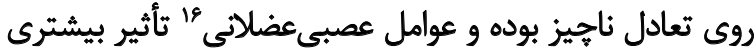

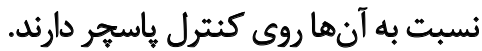

در يُورهش حاضر تفاوتهاى آمارى مشاهده شده در ميان

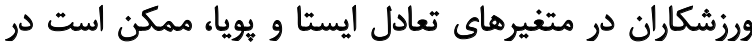

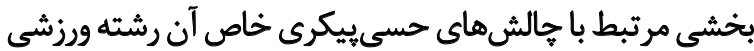

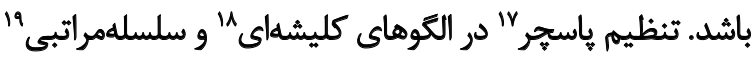
سازماندهى شده است ونياز به ادغام اطلاعات آوران از سيستهمهاى دئي

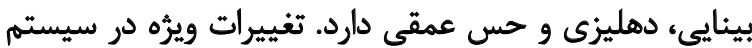

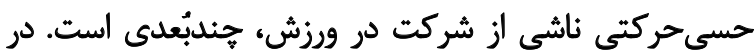

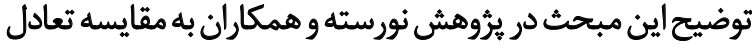

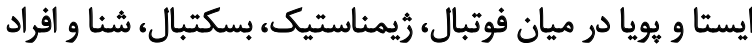

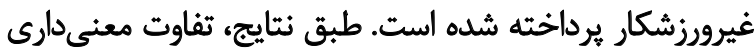

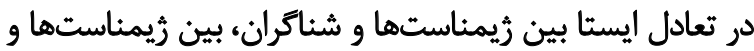

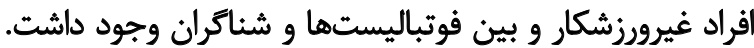

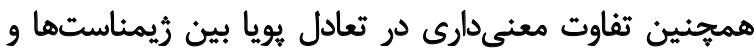

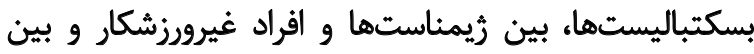

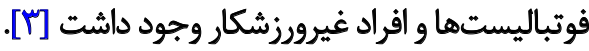

در يروهش حاضر تفاوت معنى دارى در تعادل ايستاو يويا بين

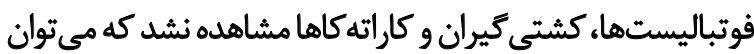

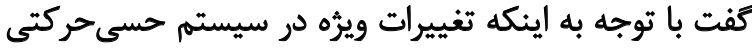

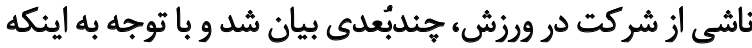

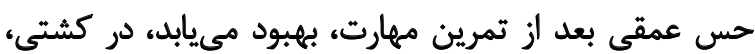

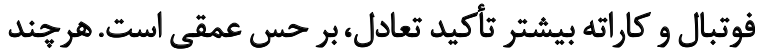

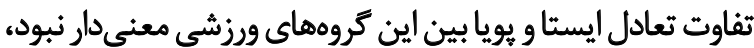

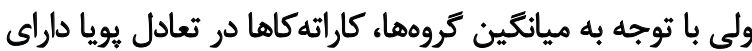

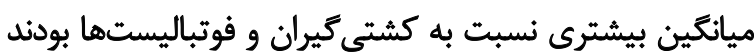

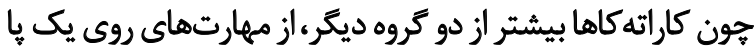

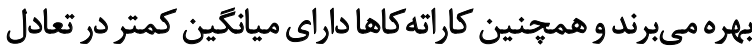

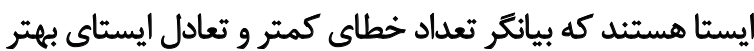

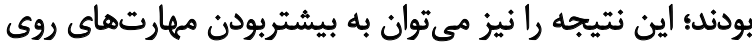

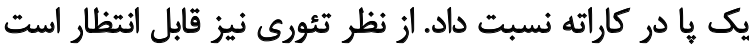

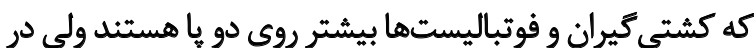

16. Neuromuscular factors

17. Postural regulation

18. Stereotypic patterns

19. Hierarchic patterns
مداخله كر بر تعادل ايستا و يويا در آزمودنىها در جدول شماره 1

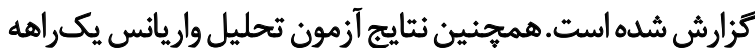

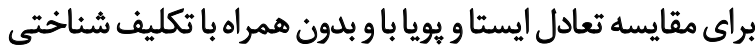

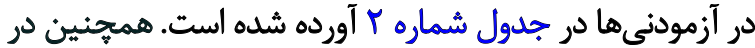

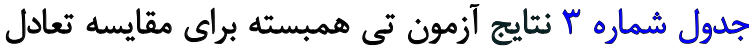

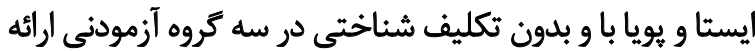

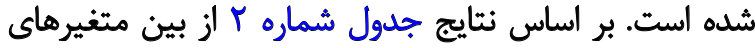

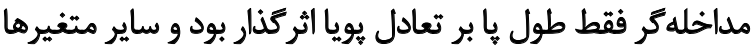
بر تعادل ايستا و يويا ثأثيرى نداشتئند

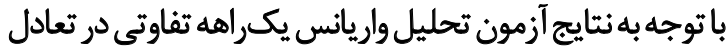

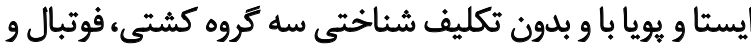

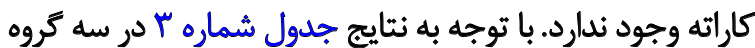

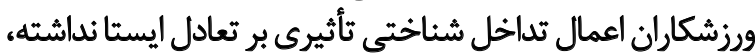

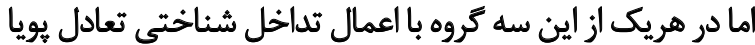

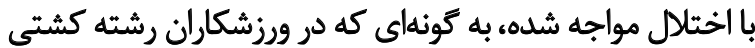

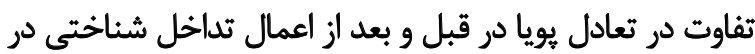

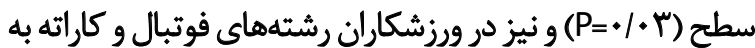

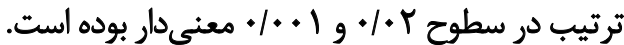

ث0

كروهى از محققين در سال هاى اخير براى بررسى نقش عوامل

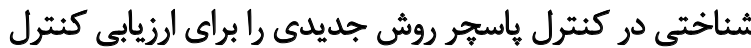

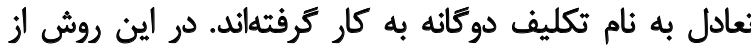

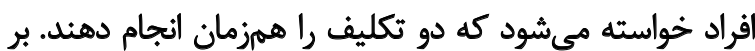

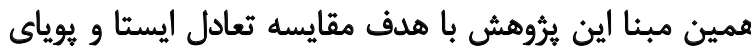

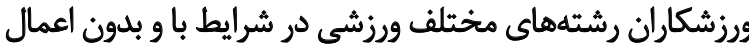

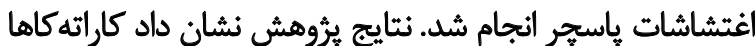

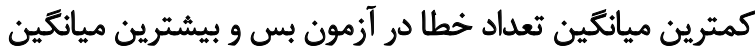

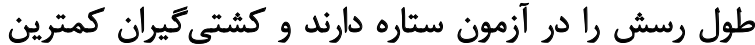

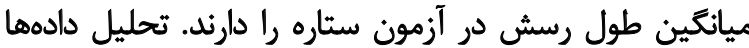

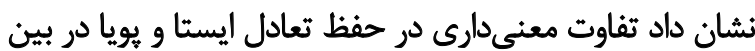

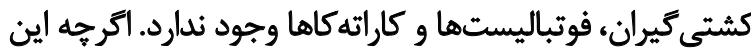

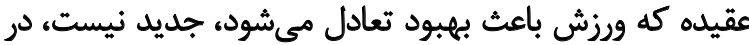

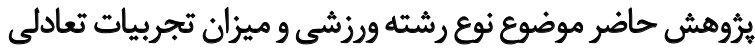

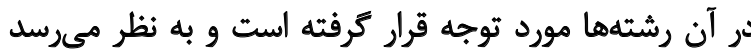

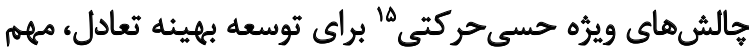

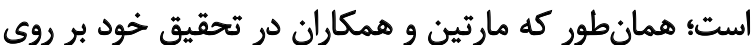

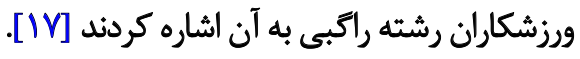

در يروهش حاضر سعى بر آن شد آزمودنىها در دامنه سنى

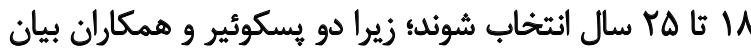

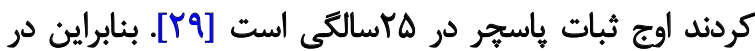

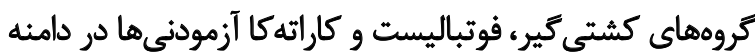

15. Specific sensorimotor challenges 
دوكانه نتايج اين كونه نبود [1/T]. در زمينه تكليف شناختى همانطور كه كفته شده ون بـان بسين

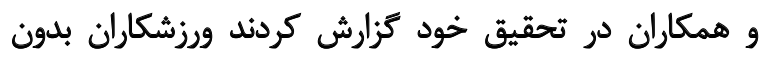

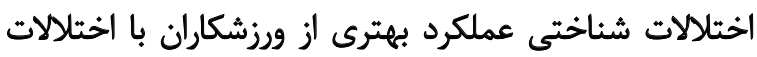

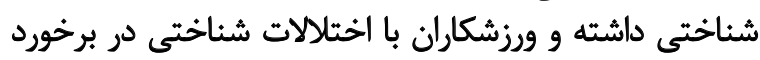

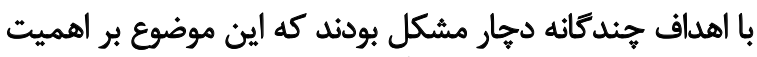

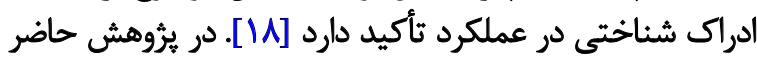

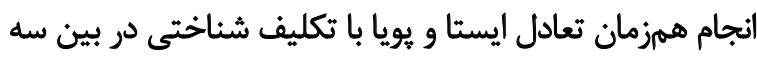

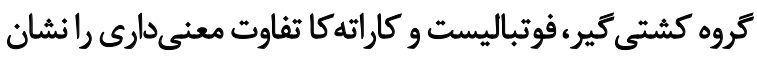

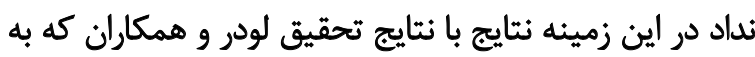

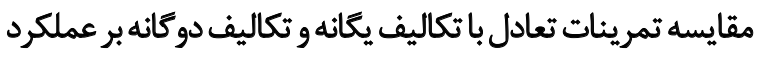

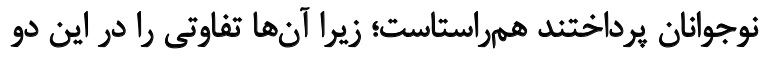

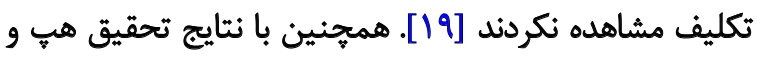

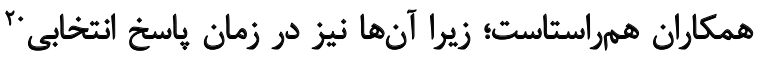

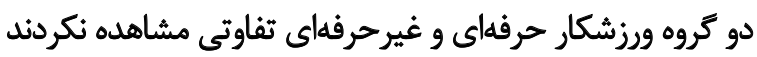

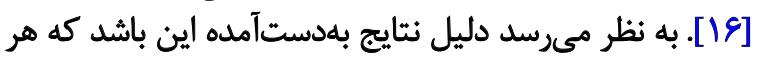

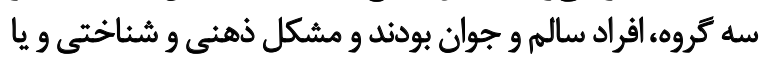

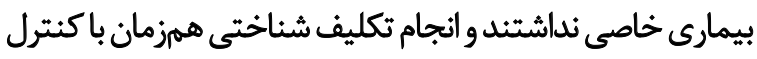

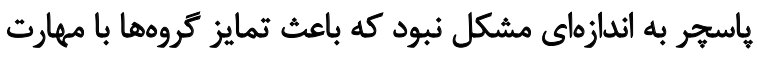

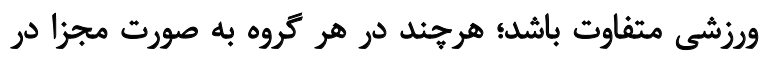

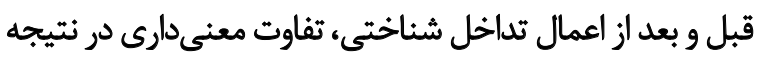

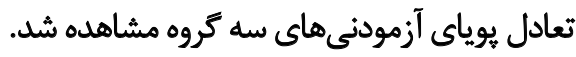

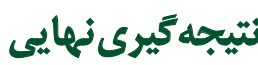

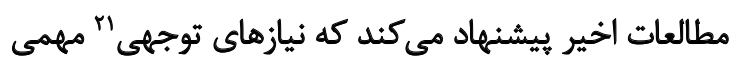

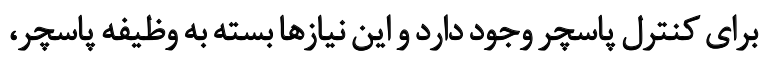

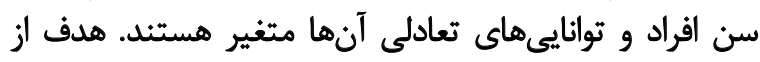

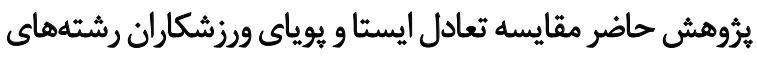

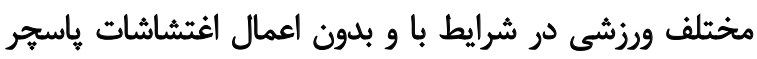

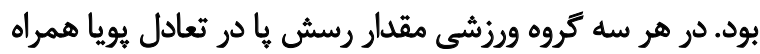

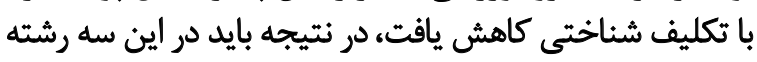

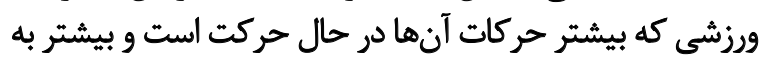

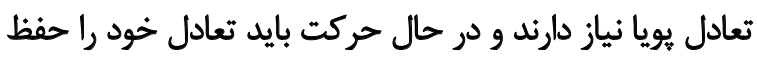

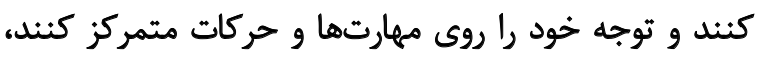

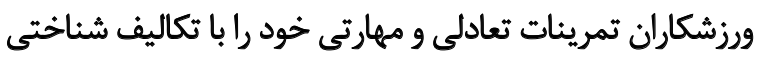

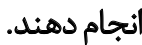

ملاحظات اخلاقي بيروى از اصول اخلاق يثوهش

تمامى آزمودنى هاى تحقيق حاضر با رضايت كامل در يُروهش

20. Choice response time

21. Attentional demands
كاراته مدتى كه روى يك يا هستند، بيشتر است. البته شايد اكر

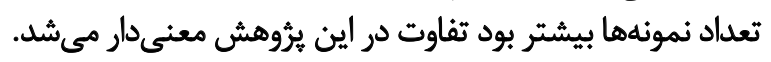

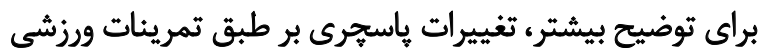

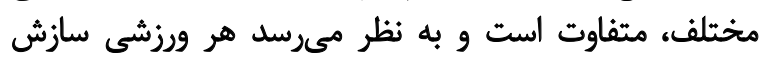

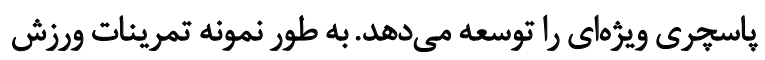

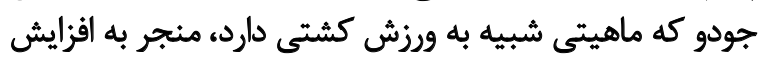

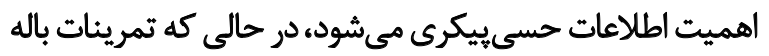

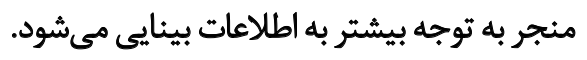

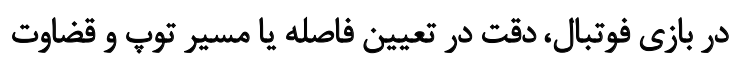

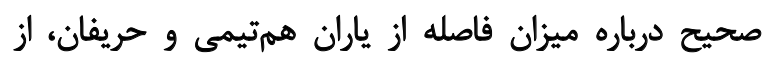

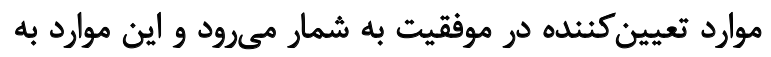

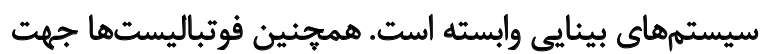

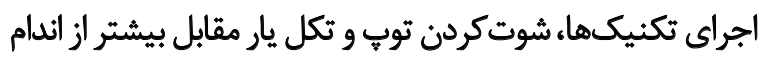

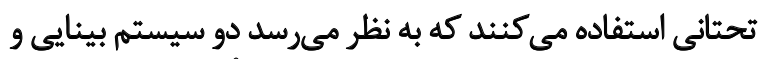

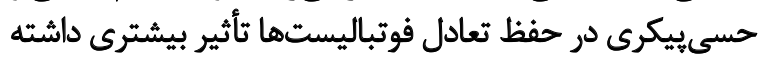

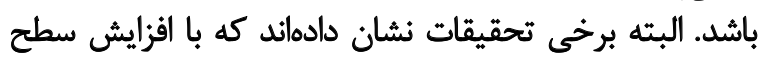

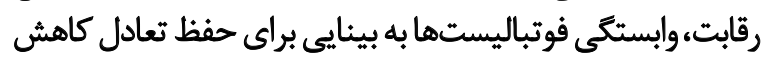
مي ريابد. بر اساس تئورى سيستمها عمل سيستم حسى در كنترل

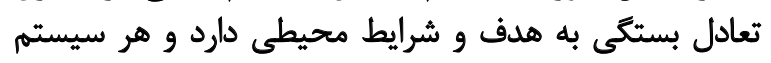

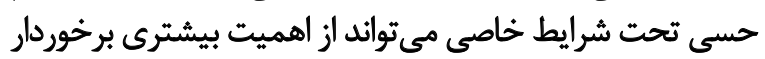

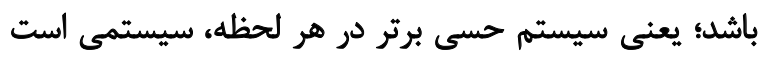

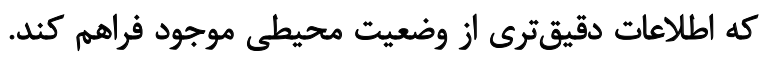

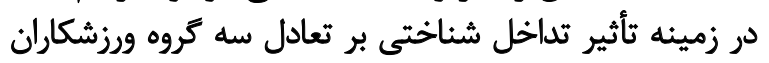

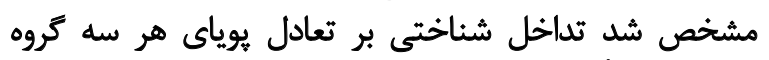

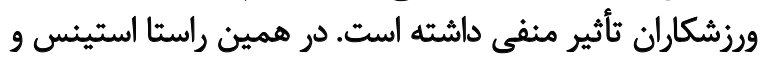

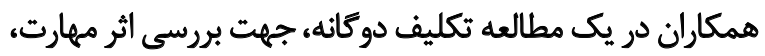

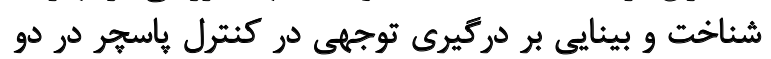

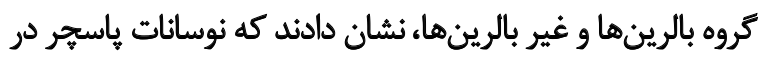

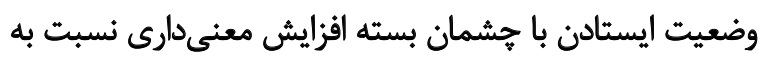

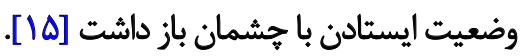

وقتى افراد در وضعيث ياسجر دشوار قرار مي ئيرند، حفظ تعادل

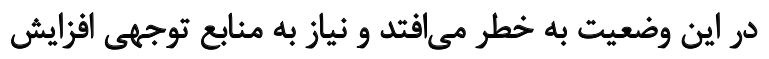

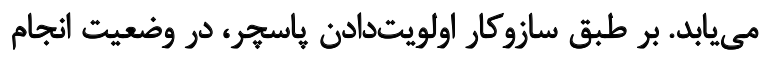

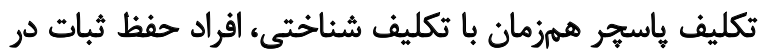

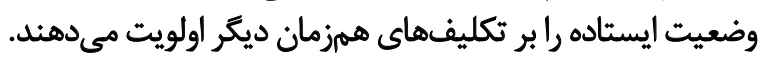

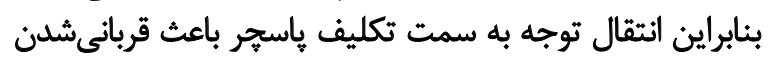

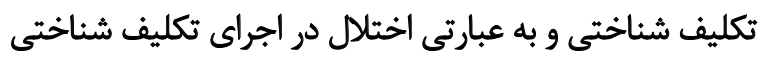

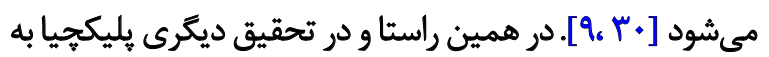

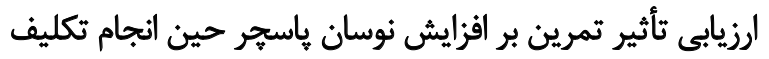

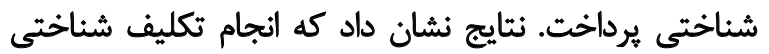

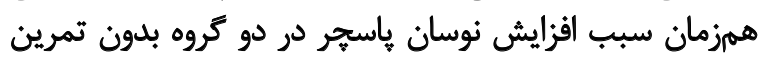
و تمرين يك تكليف شد، در صورتى كه در كروه تمرين ترون بدرن تمريف 
شركت كرده و از تمامى مراحل تحقيق آكاه بودند. همجينين با

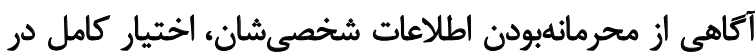
خروج از هر يك از مراحل تحقيق را داشتند.

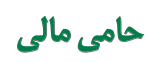

اين مقاله مستخرج از ياياننامه آقاى مصطفى شادكائلو استاد،

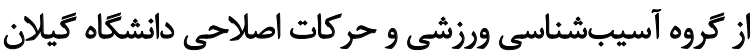
بوده و از حمايت مالى هيج بخشى استفاده نكرده است.

$$
\text { مشاركت نويسندكان }
$$

مفهومسازى: مصطفى شادكانلو و دكتر على داصغر نورسته؛

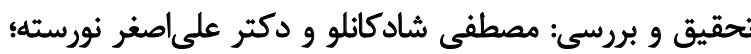

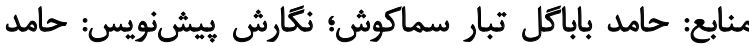

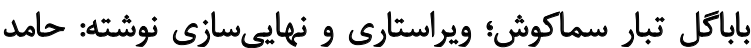

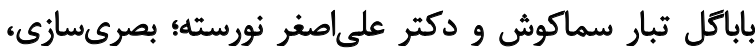
نظارت و مديريت يروزه: دكتر على اصغر نورسته

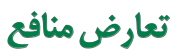
در اين مقاله هيج تعارض منافعى وجود ندارد. 


\section{References}

[1] Gautier G, Thouvarecq R, Larue J. Influence of experience on postural control: Effect of expertise in gymnastics. Journal of Motor Behavior. 2008; 40(5):400-8. [DOI:10.3200/JMBR.40.5.400-408]

[2] Gribble PA, Hertel J. Considerations for normalizing measures of the star excursion balance test. Measurement in Physical Education and Exercise Science. 2003; 7(2):89-100. [DOI:10.1207/S15327841MPEE0702_3]

[3] Norasteh AA, Mohebbi H, Shah Heydari S. [Comparison of static and dynamic balance in different athletes (Persian)]. Journal of Sport Medicine. 2011; 2(2):5-22.

[4] Hosseini Mehr SH, Norasteh AA, Khaleghi Tazji M, Abbasi A. [The effect of vibration on proprioceptive inputs of trunk muscles in healthy young males in the steering of walking (Persian)]. Journal of Sport Medicine. 2009; 1(1):25-36.

[5] Matsuda S, Demura S, Uchiyama M. Centre of pressure sway characteristics during static one-legged stance of athletes from different sports. Journal of Sports Sciences. 2008; 26(7):775-9. [DOI:10.1080/02640410701824099] [PMID]

[6] Moradi J. [Comparison of static equilibrium in soccer players and swimmers under different sensory conditions (Persian)] [MSc. thesis]. Tehran: University of Tehran; 2009

[7] Shumway-Cook A, Woollacott MH. Motor control: Translating research into clinical practice. Philadelphia: Lippincott Williams \& Wilkins; 2007.

[8] Matlin MW. Cognition. $6^{\text {th }}$ ed. Hoboken: John Wiley \& Sons; 2005.

[9] Andersson G, Hagman J, Talianzadeh R, Svedberg A, Larsen HC. Effect of cognitive load on postural control. Brain Research Bulletin. 2002; 58(1):135-9. [DOI:10.1016/S0361-9230(02)00770-0]

[10] Fraizer EV, Mitra S. Methodological and interpretive issues in posture-cognition dual-tasking in upright stance. Gait \& Posture. 2008; 27(2):271-9. [DOI:10.1016/j.gaitpost.2007.04.002] [PMID]

[11] Dault MC, Frank JS. Does practice modify the relationship between postural control and the execution of a secondary task in young and older individuals? Gerontology. 2004; 50(3):157-64. [DOI:10.1159/000076773] [PMID]

[12] Redfern MS, Jennings JR, Martin C, Furman JM. Attention influences sensory integration for postural control in older adults. Gait \& Posture. 2001; 14(3):211-6. [DOI:10.1016/S0966-6362(01)00144-8]

[13] Rahnama L, Salavati M, Akhbari B, Mazaheri M. Attentional demands and postural control in athletes with and without functional ankle instability. Journal of Orthopaedic \& Sports Physical Therapy. 2010; 40(3):180-7. [DOI:10.2519/jospt.2010.3188] [PMID]

[14] Vuillerme N, Nougier V. Attentional demand for regulating postural sway: The effect of expertise in gymnastics. Brain Research Bulletin. 2004; 63(2):161-5. [DOI:10.1016/j.brainresbull.2004.02.006] [PMID]

[15] Stins JF, Michielsen ME, Roerdink M, Beek PJ. Sway regularity reflects attentional involvement in postural control: Effects of expertise, vision and cognition. Gait \& Posture. 2009; 30(1):106-9. [DOI:10.1016/j.gaitpost.2009.04.001] [PMID]

[16] Heppe H, Kohler A, Fleddermann MT, Zentgraf K. The relationship between expertise in sports, visuospatial, and basic cognitive skills. Frontiers in Psychology. 2016; 7:904. [DOI:10.3389/fpsyg.2016.00904] [PMID] [PMCID]

[17] Martín A, Sfer AM, D’Urso Villar MA, Barraza JF. Position affects performance in multiple-object tracking in rugby union players. Frontiers in Psychology. 2017; 8:1494. [DOI:10.3389/fpsyg.2017.01494] [PMID] [PMCID]

[18] Van Biesen D, Jacobs L, McCulloch K, Janssens L, Vanlandewijck YC. Cognitive-motor dual-task ability of athletes with and without intellectual impairment. Journal of Sports Sciences. 2018; 36(5):513-21. [DOI:1 0.1080/02640414.2017.1322215] [PMID]

[19] Lüder B, Kiss R, Granacher U. Single-and dual-task balance training are equally effective in youth. Frontiers in Psychology. 2018; 9:912. [DOI:10.3389/fpsyg.2018.00912] [PMID] [PMCID]

[20] Schwesig R, Kluttig A, Leuchte S, Becker S, Schmidt H, Esperer HD. [The impact of different sports on posture regulation (German)]. Sportverletzung Sportschaden. 2009; 23(3):148-54. [DOI:10.1055/s-0028-1109576] [PMID]

[21] Leong HT, Fu SN, Ng GY, Tsang WW. Low-level taekwondo practitioners have better somatosensory organisation in standing balance than sedentary people. European Journal of Applied Physiology. 2011; 111(8):1787-93. [DOI:10.1007/s00421-010-1798-7] [PMID]

[22] Lohmann TG, Roche AF, Martorell R, editors. Anthropometric standardization reference manual. Champaign: Human Kinetics Books; 1988.

[23] Ismaila OS. Anthropometric data of hand, foot and ear of university students in Nigeria. Leonardo Journal of Sciences. 2009; (15):15-20.

[24] Cote KP, Brunet ME, Gansneder BM, Shultz SJ. Effects of pronated and supinated foot postures on static and dynamic postural stability. Journal of Athletic Training. 2005; 40(1):41-6. [PMID] [PMCID]

[25] Allen MK, Glasoe WM. Metrecom measurement of navicular drop in subjects with anterior cruciate ligament injury. Journal of Athletic Training. 2000; 35(4):403-6. [PMID] [PMCID]

[26] Bressel E, Yonker JC, Kras J, Heath EM. Comparison of static and dynamic balance in female collegiate soccer, basketball, and gymnastics athletes. Journal of Athletic Training. 2007; 42(1):42-6. [PMID] [PMCID]

[27] Smith BI, Docherty CL, Simon J, Klossner J, Schrader J. Ankle strength and force sense after a progressive, 6-week strength-training program in people with functional ankle instability. Journal of Athletic Training 2012; 47(3):282-8. [DOI:10.4085/1062-6050-47.3.06] [PMID] [PMCID]

[28] Andersson G, Hagman J, Talianzadeh R, Svedberg A, Larsen HC. Dual-task study of cognitive and postural interference in patients with vestibular disorders. Otology \& Neurotology. 2003; 24(2):289-93. [DOI:10.1097/00129492-200303000-00026] [PMID]

[29] Du Pasquier RA, Blanc Y, Sinnreich M, Landis T, Burkhard P, Vingerhoets FJG. The effect of aging on postural stability: A cross sectional and longitudinal study. Neurophysiologie Clinique/Clinical Neurophysiology. 2003; 33(5):213-8. [DOI:10.1016/j.neucli.2003.09.001] [PMID]

[30] Riley MA, Baker AA, Schmit JM. Inverse relation between postura variability and difficulty of a concurrent short-term memory task. Brain Research Bulletin. 2003; 62(3):191-5. [DOI:10.1016/j.brainresbull.2003.09.012] [PMID]

[31] Pellecchia GL. Dual-task training reduces impact of cognitive task on postural sway. Journal of Motor Behavior. 2005; 37(3):239-46. [DOI:10.3200/JMBR.37.3.239-246] [PMID] 